\title{
Fiber-Optic Distributed Sensor Based on Hybrid Raman and Brillouin Scattering Employing Multiwavelength Fabry-Pérot Lasers
}

\author{
Gabriele Bolognini, Member, IEEE, Marcelo A. Soto, and Fabrizio Di Pasquale, Member, IEEE
}

\begin{abstract}
We propose the use of standard multiwavelength Fabry-Pérot (FP) lasers for distributed strain and temperature measurement in hybrid Raman- and Brillouin-based fiber-optic sensors. The multiple longitudinal modes of FP lasers allow for accurate spontaneous Raman scattering measurement and for simultaneous high-sensitivity coherent detection of the Brillouin frequency shift parameter for all longitudinal modes. Experimental results confirm great performance improvement in simultaneous strain and temperature measurement using a single optical fiber.
\end{abstract}

Index Terms-Brillouin scattering, laser modes, optical fiber measurements, optical time-domain reflectometry (OTDR).

\section{INTRODUCTION}

D ISTRIBUTED fiber-optic sensors, for simultaneous temperature and strain measurement, find several practical applications, ranging from structural-health monitoring of large structures, to leakage and strain detection in power cables and pipelines, from early warning of landslides to borehole and underground mine monitoring. Most distributed fiber-optic sensors for simultaneous strain and temperature measurement are currently based on Brillouin scattering measurement [1]. One of the main issues to be addressed in such systems is related to the strain and temperature cross-sensitivity of the Brillouin frequency shift (BFS), which is often overcome in practical systems by using two different sensing fibers, only one of them embedded in the structure to be monitored.

Simultaneous strain and temperature sensing based on a single optical fiber, however, can be implemented using either hybrid Raman-Brillouin scattering detection [2] or simultaneous detection of both Brillouin intensity and BFS [3]. Although both schemes allow us in principle to distinguish between strain and temperature, several drawbacks limit their practical applications [2], [4]. In hybrid Raman-Brillouin schemes, the measured spontaneous Raman-scattered anti-Stokes line, which is strain-independent, is used to estimate the fiber temperature and also to correct the temperature-dependence of the spontaneous BFS parameter, providing reliable, temperature-independent strain measurements. However, the noise in the anti-Stokes Raman scattering

Manuscript received April 02, 2009; revised July 01, 2009. Current version published September 30, 2009

The authors are with Scuola Superiore Sant'Anna, Pisa 56124, Italy (e-mail: gabriele.bolognini@sssup.it; m.soto@sssup.it; fabrizio.dipasquale@sssup.it).

Color versions of one or more of the figures in this letter are available online at http://ieeexplore.ieee.org.

Digital Object Identifier 10.1109/LPT.2009.2028899 trace, impacting the temperature estimation, has been identified as the major inaccuracy source in simultaneous temperature-strain measurements with hybrid sensing [2]. Moreover, in systems based on optical time-domain reflectometry (OTDR), commonly used in hybrid sensing, the achievable spatial resolution depends proportionally on the pulsewidth, leading to a well-known trade-off between spatial resolution and measurement accuracy. In order to overcome these performance limitations, high input pump power levels should be used, which are, however, incompatible with the onset on nonlinear effects, such as stimulated Raman and Brillouin scattering. On the other hand, the narrow Brillouin gain bandwidth (few tens of megahertz in silica fibers) requires the use of narrowband optical sources for accurate BFS measurement, such as distributed-feedback (DFB) lasers or external cavity lasers; such lasers are characterized by a typical output power below few tens of milliwatts, which is not really effective for Raman-based temperature-sensing. It is then evident that the optical sources to be used in hybrid Raman-Brillouin sensors play a key role in dictating their performance and must be properly selected.

In this letter, we propose and characterize, for first time to our knowledge, the use of multiwavelength Fabry-Pérot (FP) lasers for hybrid Raman-Brillouin distributed strain and temperature sensors. We show that FP lasers, when suitably used within OTDR-based schemes and coherent detection, allow for a great accuracy enhancement in both temperature and strain measurements. In the proposed scheme, the simultaneous measurement of the BFS parameter for all FP longitudinal modes is performed in one step by using a single photodetector and a self-heterodyne detection scheme.

\section{THEORY}

In hybrid Raman-Brillouin sensors for temperature and strain measurement, the spontaneous Raman and Brillouin scattered lights from the same optical fiber are simultaneously detected. The temperature distribution, based on Raman scattering, is commonly derived from the anti-Stokes $\left(I_{\mathrm{AS}}\right)$ to the Rayleigh-backscattering $\left(I_{\mathrm{BS}}\right)$ light power ratio, which is dependent on the absolute temperature $T$ according to

$$
\frac{I_{\mathrm{AS}}}{I_{\mathrm{BS}}} \propto\left[\exp \left(\frac{h \Delta \nu_{R}}{k T}\right)-1\right]^{-1}
$$

where $h$ is the Planck constant, $k$ is the Boltzmann constant, and $\Delta \nu_{R}$ is the separation between Raman anti-Stokes and pump light frequencies. The strain variation $\Delta \varepsilon$ is derived from the 


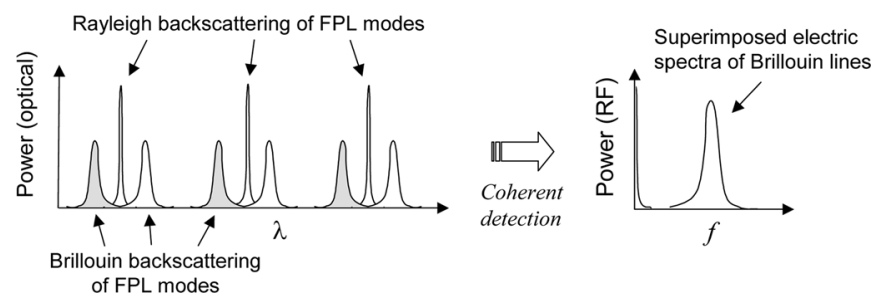

Fig. 1. Principle diagram indicating the features of optical and electric spectra of FP laser scattering light under a coherent detection scheme.

BFS parameter $\Delta \nu_{B}$, which linearly depends on both strain and temperature according to the formula

$$
\Delta \nu_{B}=C_{\nu_{B} \varepsilon} \cdot \Delta \varepsilon+C_{\nu_{B} T} \cdot \Delta T
$$

where $C_{\nu B \varepsilon}=0.048 \mathrm{MHz} / \mu \varepsilon$ and $C_{\nu B T}=1.07 \mathrm{MHz} /{ }^{\circ} \mathrm{C}$ are the strain and temperature coefficients for BFS in silica fibers [4]. The temperature contribution in (2) is obtained from the Raman measurement based on (1), thus providing a temperature-independent estimation of the strain variation $\Delta \varepsilon$.

FP semiconductor lasers, characterized by a grid of narrowband longitudinal modes exhibiting a typical frequency difference of a few tens of gigahertz, and a full-width at half-maximum (FWHM) bandwidth of several nanometers, can be effectively exploited for distributed Raman sensing. In fact their high available power levels (within the range 20-26 dBm) and large bandwidth allow for accurate measurement of both Raman anti-Stokes and Rayleigh components, also avoiding coherent-Rayleigh noise (CRN) issues [5].

When an FP laser is coupled into a single-mode optical fiber for Raman-sensing purposes, each longitudinal mode also generates spontaneous Stokes and anti-Stokes Brillouin backscattering. The FWHM of each FP laser mode is narrower than the Brillouin spectrum, and the spectral separation among adjacent modes in an FP laser is generally larger than twice the BFS in silica; this implies that it is possible to clearly identify the Brillouin-scattered Stokes and anti-Stokes lights from each longitudinal mode of a typical FP laser.

It is then very interesting to investigate the possibility to measure the BFS parameter for all FP longitudinal modes with a single photodetector, using a optical self-heterodyne detection scheme in which the optical local oscillator (OLO) is generated by splitting a portion of the same FP laser light. In such a scheme, whose principle of operation is represented in Fig. 1, the Brillouin-scattering light originating from each mode will interfere with all FP longitudinal modes. Thus, these beatings will generate in particular a strong photocurrent component at around $10.9 \mathrm{GHz}$ for silica fibers, which corresponds to the average Brillouin scattering spectrum for all modes (i.e., the sum of the beatings of each FP longitudinal mode with its Brillouin-scattered lines). Provided that the FP laser light is sufficiently stable in wavelength within a single trace acquisition (e.g., through accurate laser temperature control), this novel optical coherent detection scheme, based on multi- $\lambda$ OLO combined with direct Raman scattering detection, can thus lead to great performance improvement in simultaneous strain and temperature measurement with respect to the use of a single narrowband optical source, such as a DFB laser [2]. Moreover, due to

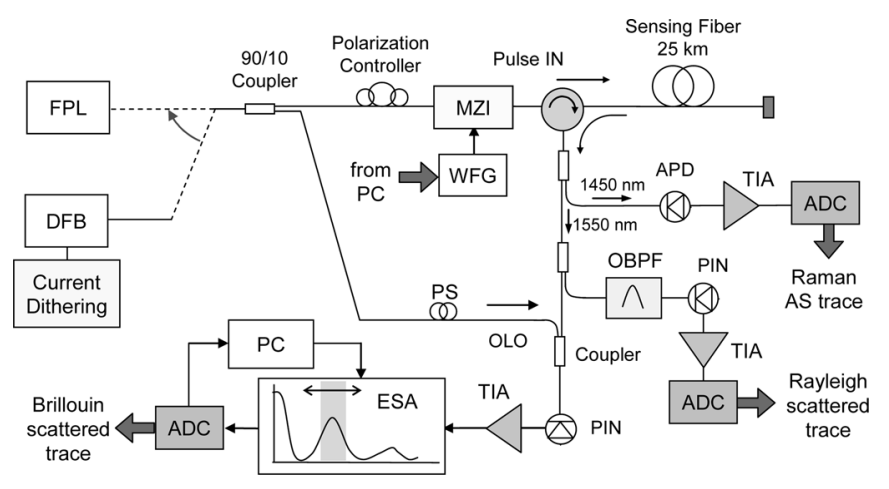

Fig. 2. Experimental setup of hybrid Raman-Brillouin distributed strain and temperature sensor employing FP lasers.

the low peak power of each FP longitudinal mode, the possible pulse coding [6] with this kind of optical sources is expected to further enhance the receiver performance in both Raman intensity and BFS measurements, also avoiding potential performance degradation due to the influence of coding in the stimulated Brillouin threshold [6].

\section{EXPERIMENTAL SETUP}

The experimental setup we have used is schematically described in Fig. 2; DFB and FP lasers are alternatively employed in order to compare their sensing performances. The output power of the used DFB laser at $1550 \mathrm{~nm}$ is $8.5 \mathrm{dBm}$, allowing for $P_{\mathrm{IN}}=1.5 \mathrm{dBm}$ at the sensing fiber input. Note that due to the DFB narrowband characteristic, current dithering is required when measuring the Rayleigh component in order to reduce CRN. On the other hand, the output power of the used FP laser at around $1550 \mathrm{~nm}$ is $22 \mathrm{dBm}$, ensuring $P_{\mathrm{IN}}=15 \mathrm{dBm}$ at the sensing fiber input. Moreover, the broadband spectrum of the multimode FP laser allows for inherent wavelength averaging, effectively suppressing CRN during Rayleigh measurements. The continuous-wave light of the source is split into two parts using a 10/90 optical coupler: $10 \%$ of the light is used as OLO at the receiver and the other $90 \%$ is modulated with 350 -ns single pulses, allowing for $35-\mathrm{m}$ spatial resolution. Optical pulses are sent along $25 \mathrm{~km}$ of standard single-mode fiber (SSMF), and the backscattered light is coupled into the receiver side, which is composed of three stages. The first stage (Raman receiver) selects the light band at around $1450 \mathrm{~nm}$, so that the anti-Stokes Raman component can be detected by an avalanche photodiode (APD), followed by a transimpedance amplifier (TIA) and an analog-to-digital converter (ADC) connected to a computer (PC). The second stage (Rayleigh receiver) consists of a direct-detection receiver used for Rayleigh-backscattering measurement at around $1550 \mathrm{~nm}$; it is composed of a PIN, followed by a TIA and another ADC connected to the PC. The third stage (Brillouin receiver) is a heterodyne receiver (optical and electrical), where the OLO is mixed with the Brillouin components backscattered by the $25 \mathrm{~km}$ of sensing fiber. An electrical spectrum analyzer (ESA) in zero-span mode is employed within electric heterodyne scheme for trace acquisition in Brillouin spectrum measurements. A polarization scrambler (PS) is used to depolarize the OLO in order to reduce polarization-induced noise produced in coherent detectors. The PS is implemented by using a polarization controller and $10 \mathrm{~m}$ 


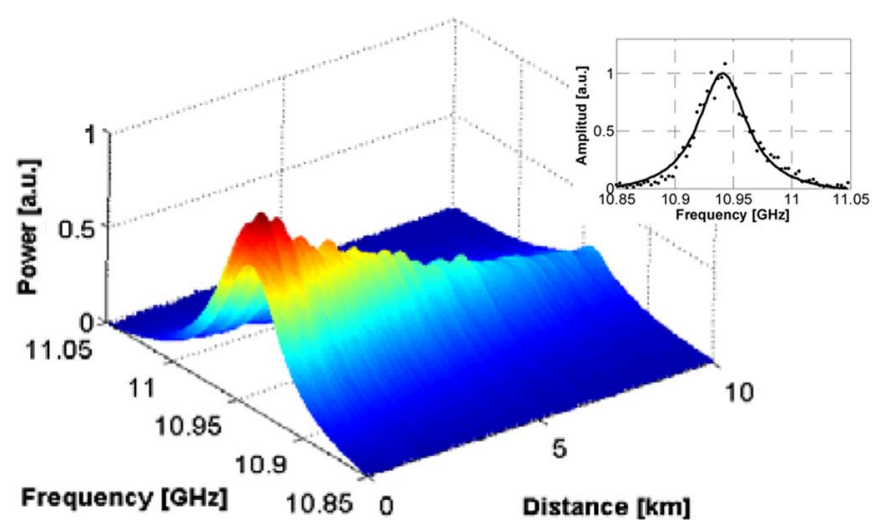

Fig. 3. Brillouin spectrum as a function of the distance. Inset: Lorentzian fitting to the measured data points (at 5-km distance).

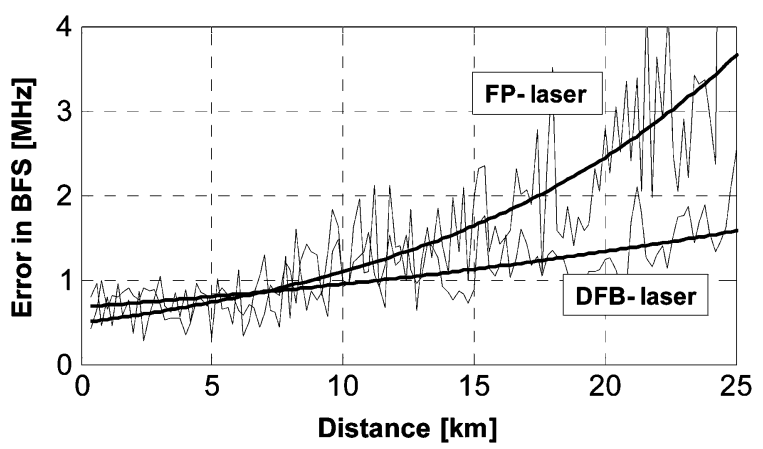

Fig. 4. Error in BFS versus distance, for both DFB and FP laser.

of polarization-maintaining fiber (PMF), reducing the degree of polarization of the FP laser down to less than $1 \%$.

\section{RESULTS}

The FP laser used in the experiment is characterized by $\sim 30$ longitudinal modes $(\sim 7$-dBm power per mode, wavelength separation $\sim 0.3 \mathrm{~nm}$ ), each one generating a couple of Stokes and anti-Stokes Brillouin components. The same laser is also used as multi- $\lambda$ OLO at the PIN photodiode producing an electrical signal within the Brillouin gain bandwidth, at $\sim 10.9 \mathrm{GHz}$, representing the summed contribution of Stokes and anti-Stokes components generated by all FP modes.

To estimate the average BFS, a least-squares algorithm has been used to fit the measured spectrum with a Lorentzian curve at each fiber position. The obtained spectrum as a function of the distance is shown in Fig. 3, together with a fitting of the measured spectrum at 5-km distance (Fig. 3 inset). Fig. 4 compares the experimental BFS root-mean-square errors versus distance for both DFB and FP lasers (after $150 \mathrm{k}$ averages). It is clearly evident that FP laser-based measurements are affected by stronger noise levels. This is likely due to optical noise sources, such as the BFS dependence on the mode wavelength and the frequency drift of the FP multilongitudinal modes within the trace acquisition time $(250 \mu \mathrm{s})$. However, such noise sources have only a small impact on the ultimate temperature and strain resolutions of the hybrid sensor, reported versus sensing distance in Figs. 5 and 6, which are significantly improved by the use of FP lasers. The limiting factor in hybrid sensing is in fact essentially given by the noise in the anti-Stokes Raman intensity measurement, which is greatly reduced when using

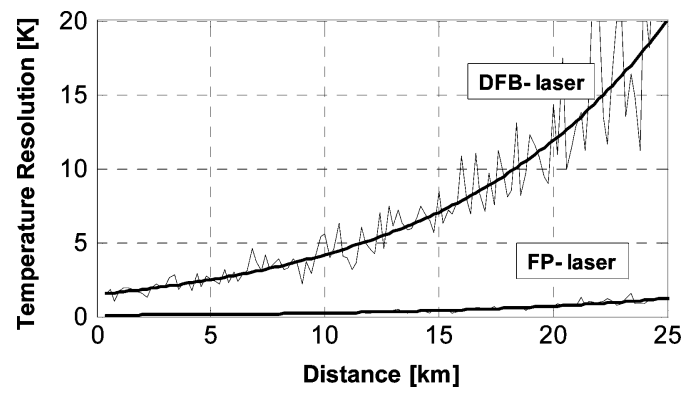

Fig. 5. Temperature resolution versus distance, for both DFB and FP lasers.

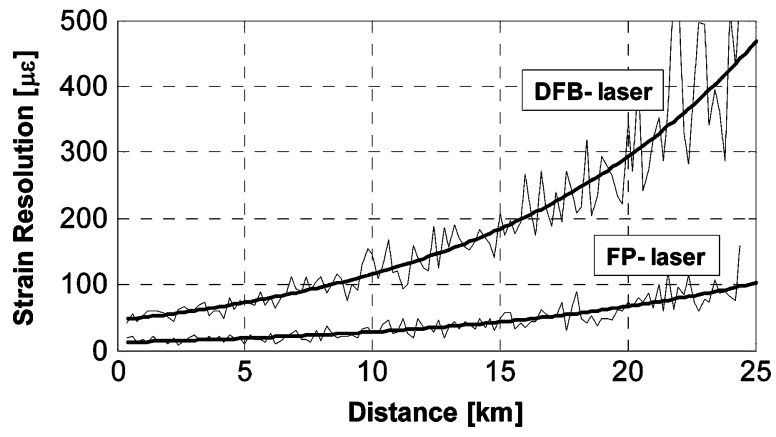

Fig. 6. Strain resolution versus distance, for both DFB and FP lasers.

higher power FP lasers. The achieved temperature resolution, shown in Fig. 5, is reduced from $20 \mathrm{~K}$ down to $\sim 1.2 \mathrm{~K}$ at 25-km distance when using the FP laser-based technique. The accuracy enhancement in the anti-Stokes Raman intensity also has a significant impact on the final strain resolution which is reduced from $\sim 470 \mu \varepsilon$ down to $\sim 100 \mu \varepsilon$ at $25-\mathrm{km}$ distance (Fig. 6). Also note that for a sensor performance analysis it is not required to apply any actual strain or temperature change to the fiber, since the attainable resolution parameters depend ultimately on the signal-to-noise ratio of the measured traces.

In conclusion, we have shown that the use of multiwavelength FP laser sources can effectively enhance the performance of hybrid Raman-Brillouin-based sensor for simultaneous strain and temperature measurement. Further improvements are expected by applying optical pulse coding and by using more stable multi- $\lambda$ laser sources.

\section{REFERENCES}

[1] T. Horiguchi, K. Shimizu, T. Kurashima, M. Tateda, and Y. Koyamada, "Development of a distributed sensing technique using Brillouin scattering," J. Lightw. Technol., vol. 13, no. 7, pp. 1296-1301, Jul. 1995.

[2] M. N. Alahbabi, Y. T. Cho, and T. P. Newson, "Simultaneous temperature and strain measurement with combined spontaneous Raman and Brillouin scattering," Opt. Lett., vol. 30, no. 11, pp. 1276-1278, 2005.

[3] S. M. Maughan, H. Kee, and T. P. Newson, "Simultaneous distributed fibre temperature and strain sensor using microwave coherent detection of spontaneous Brillouin backscatter," Meas. Sci. Technol., vol. 12, pp. 834-842, 2001.

[4] R. Bernini, A. Minardo, and L. Zeni, "An accurate high-resolution technique for distributed sensing based on frequency-domain Brillouin scattering," IEEE Photon. Technol. Lett., vol. 18, no. 1, pp. 280-282, Jan. 1, 2006.

[5] K. De Souza, "Significance of coherent Rayleigh noise in fibre-optic distributed temperature sensing based on spontaneous Brillouin scattering," Meas. Sci. Technol., vol. 17, pp. 1065-1069, 2006.

[6] M. A. Soto, P. K. Sahu, G. Bolognini, and F. Di Pasquale, "Brillouinbased distributed temperature sensor employing pulse coding," IEEE Sensors J., vol. 8, no. 3, pp. 225-226, Mar. 2008. 